\title{
Active-Passive Control Strategy for Adjacent Buildings
}

\author{
Francisco Palacios-Quiñonero, Josep M. Rossell, José Rodellar, and Hamid R. Karimi
}

\begin{abstract}
In this paper, a control strategy to mitigate the vibrational response of adjacent buildings under seismic excitation is presented. The proposed strategy combines interbuilding passive actuators with active actuators placed in the building stories. The main ideas are presented by means of a simplified two-building model; however, a semi-decentralized overlapping approach via the inclusion principle has been used to impose a proper information exchange structure suitable for wireless control of large buildings. Numerical simulations have been carried out to assess the performance of the proposed methodology with promising results. The overall control system exhibits some degree of fault-tolerance since only partial degradation of the control performance results from partial failures of the control system.
\end{abstract}

\section{INTRODUCTION}

Over the last few decades, structural vibration control of large civil engineering structures has become an increasingly active research field. In recent years, the Connected Control Method (CCM) has been proposed as a viable means to protect adjacent flexible structures against earthquakes. In the CCM, two independent structures are linked together by coupling devices to provide appropriate reaction control forces. The application of the CCM using different kinds of passive, active, or semi-active control strategies have been investigated in a number of papers [1], [2], [3], [4], [5]; in all these works, the control actuators are located in the coupling devices

Also recently, wireless communications has made a significant impact in the area of vibrational control of large buildings [6], [7]. The use of wireless communications, instead of the classical coaxial wiring, can critically reduce the installation and maintenance costs; furthermore, it can also add flexibility to the control system, allowing the implementation of new control strategies without costly modifications. However, to improve the communications robustness and to achieve higher sampling frequencies in the real-time control operation, the controllers need to operate using local information provided by neighboring sensors. Consequently, a decentralized control approach is required for a realistic treatment of Wireless Networked Control Systems (WNCS). In this context, overlapping controllers designed via the Inclusion Principle can be a specially

This work was supported in part by the Spanish Committee for Science and Technology (CICYT) under Grants DPI2008-06699-C02 and DPI200806564-C02-02.

F. Palacios, J.M. Rossell, and J. Rodellar are with the Department of Applied Mathematics III, Universitat Politècnica de Catalunya (UPC), Spain. (francisco.palacios@upc.edu), (josep.maria.rossell@upc.edu), (jose.rodellareupc.edu),

H.R. Karimi is with the Department of Engineering, University of Adger, Norway. (hamid.r.karimi@uia.no) suitable control strategy for large-scale WNCS, reducing the design and operation computational effort and providing semi-decentralized controllers which satisfy the information exchange constraints, [8], [9].

The aim of this paper is to design a decentralized controller for large adjacent buildings suitable for wireless implementation. To this end, we consider a two-building coupled system with the following control structure: (i) Every story is equipped with an ideal active actuator, (ii) passive actuators are located at the linking elements, (iii) in each building, the active actuators are operated by an independent local controller, (iv) for large buildings, the local controller has a semi-decentralized overlapping structure, suitable for wireless implementation.

Due to the complexity of the overall system, we have chosen a minimal configuration that allows a clear presentation of the main ideas while maintaining the generality of the approach. This configuration consists of a three-story building, which is considered as a large building, linked to a two-story building. For this system, local controllers have been independently designed: a standard LQR state-feedback controller for the two-story building, and an overlapping state-feedback LQR controller for the three-story building. The linking passive actuators have been modeled as viscoelastic dampers. A centralized state-feedback LQR control for the overall system, which has been taken as a reference in the performance assessment, has also been computed. Numerical simulations have been conducted to assess the performance of the proposed methodology.

The paper is organized as follows. In Section 2, a simplified model of the two-building coupled system is provided. Section 3 presents a brief summary relative to the Inclusion Principle. In Section 4, the decentralized overlapping controller and the reference centralized controller are computed. Finally, in Section 5, numerical simulations of the free and controlled responses are presented and compared.

\section{TWO-BUILDING COUPLED MODEL}

In this section, a simplified mechanical model for a twobuilding coupled system formed by a three-story building and a two-story building linked by viscoelastic dampers is presented.The buildings motion can be described by

$$
\mathbf{M} \ddot{q}(t)+\mathbf{C} \dot{q}(t)+\mathbf{K} q(t)=\mathbf{T}_{\mathbf{u}} u(t)-\mathbf{M} \mathbf{T}_{\mathbf{w}} \omega(t),
$$

where $\mathbf{M}$ is the mass matrix; $\mathbf{K}$, and $\mathbf{C}$ are the total stiffness and damping matrices, respectively, including the buildings stiffness and damping coefficients as well as the stiffness and damping coefficients of the viscoelastic dampers; $q(t)$ is the vector of relative displacements with respect to the 
ground; $u(t)$ is the vector of control forces, and $\mathbf{T}_{\mathbf{u}}=\mathbf{I}_{5 \times 5}$ is the control location matrix; $\mathbf{T}_{\mathbf{w}}$ is the index vector with all its elements equal to 1 , and $\omega(t)$ is the ground acceleration (see Fig. 1).

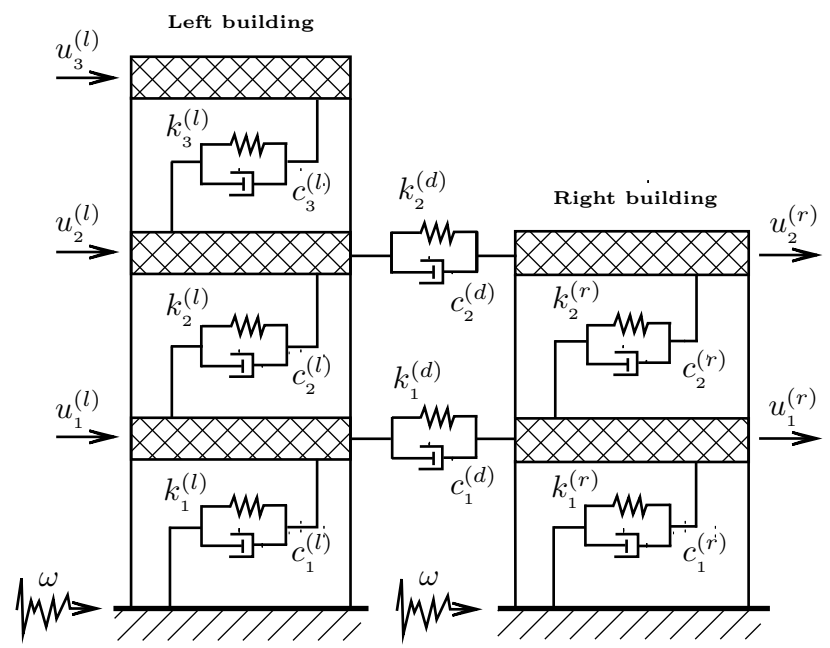

Fig. 1. Structural model for adjacent buildings

With the notations indicated in Fig. 1, the matrices in equation (1) have the following structure:

$$
\begin{aligned}
& \mathbf{M}=\operatorname{diag}\left[m_{1}^{(l)}, m_{2}^{(l)}, m_{3}^{(l)}, m_{1}^{(r)}, m_{2}^{(r)}\right], \\
& \mathbf{C}=\mathbf{C}^{\mathbf{s}}+\mathbf{C}^{\mathbf{d}}, \quad \mathbf{K}=\mathbf{K}^{\mathbf{s}}+\mathbf{K}^{\mathbf{d}},
\end{aligned}
$$

where the story damping matrix is

$$
\mathbf{C}^{\mathbf{s}}=\left[\begin{array}{cc}
\mathbf{C}_{\mathbf{L}} & \mathbf{0} \\
\mathbf{0} & \mathbf{C}_{\mathbf{R}}
\end{array}\right] \text {, }
$$

with

$$
\mathbf{C}_{\mathbf{L}}=\left[\begin{array}{ccc}
c_{1}^{(l)}+c_{2}^{(l)} & -c_{2}^{(l)} & 0 \\
-c_{2}^{(l)} & c_{2}^{(l)}+c_{3}^{(l)} & -c_{3}^{(l)} \\
0 & -c_{3}^{(l)} & c_{3}^{(l)}
\end{array}\right], \mathbf{C}_{\mathbf{R}}=\left[\begin{array}{cc}
c_{1}^{(r)}+c_{2}^{(r)} & -c_{2}^{(r)} \\
-c_{2}^{(r)} & c_{2}^{(r)}
\end{array}\right] .
$$

The stiffness matrix of the adjacent buildings is

$$
\mathbf{K}^{\mathbf{s}}=\left[\begin{array}{cc}
\mathbf{K}_{\mathbf{L}} & \mathbf{0} \\
\mathbf{0} & \mathbf{K}_{\mathbf{R}}
\end{array}\right]
$$

with

$$
\mathbf{K}_{\mathbf{L}}=\left[\begin{array}{ccc}
k_{1}^{(l)}+k_{2}^{(l)} & -k_{2}^{(l)} & 0 \\
-k_{2}^{(l)} & k_{2}^{(l)}+k_{3}^{(l)} & -k_{3}^{(l)} \\
0 & -k_{3}^{(l)} & k_{3}^{(l)}
\end{array}\right], \mathbf{K}_{\mathbf{R}}=\left[\begin{array}{cc}
k_{1}^{(r)}+k_{2}^{(r)} & -k_{2}^{(r)} \\
-k_{2}^{(r)} & k_{2}^{(r)}
\end{array}\right] .
$$

The damping matrix of the viscoelastic dampers is

$$
\mathbf{C}^{\mathbf{d}}=\left[\begin{array}{ccccc}
c_{1}^{(d)} & 0 & 0 & -c_{1}^{(d)} & 0 \\
0 & c_{2}^{(d)} & 0 & 0 & -c_{2}^{(d)} \\
0 & 0 & 0 & 0 & 0 \\
-c_{1}^{(d)} & 0 & 0 & c_{1}^{(d)} & 0 \\
0 & -c_{2}^{(d)} & 0 & 0 & c_{2}^{(d)}
\end{array}\right],
$$

and the stiffness matrix of the viscoelastic dampers is

$$
\mathbf{K}^{\mathbf{d}}=\left[\begin{array}{ccccc}
k_{1}^{(d)} & 0 & 0 & -k_{1}^{(d)} & 0 \\
0 & k_{2}^{(d)} & 0 & 0 & -k_{2}^{(d)} \\
0 & 0 & 0 & 0 & 0 \\
-k_{1}^{(d)} & 0 & 0 & k_{1}^{(d)} & 0 \\
0 & -k_{2}^{(d)} & 0 & 0 & k_{2}^{(d)}
\end{array}\right] .
$$

From the second-order model (1), a first-order state space model can be derived

$$
\begin{aligned}
\mathbf{S}: \dot{x}(t) & =A x(t)+B u(t)+E w(t), \\
y(t) & =C_{y} x(t),
\end{aligned}
$$

where the state vector $x(t) \in \mathbb{R}^{10}$ groups together the displacements and the velocities arranged in increasing order, that is,

$$
x(t)=\left[q_{1}(t), \dot{q}_{1}(t), \ldots, q_{5}(t), \dot{q}_{5}(t)\right]^{T},
$$

where $q_{i}(t)=q_{i}^{(l)}(t), i=1,2,3$, is the displacement relative to the ground of the $i$ th story in the left building, and $q_{4}(t)=q_{1}^{(r)}(t), q_{5}(t)=q_{2}^{(r)}(t)$ denote the corresponding ones for the right building. The matrices of the state-space model used in the controllers design and the response numerical simulations are

$$
\begin{aligned}
& A=\left[\begin{array}{cccccccccc}
0 & 1 & 0 & 0 & 0 & 0 & 0 & 0 & 0 & 0 \\
-6201 & -0.2 & 3100 & 0.1 & 0 & 0 & 0 & 0 & 0 & 0 \\
0 & 0 & 0 & 1 & 0 & 0 & 0 & 0 & 0 & 0 \\
3100 & 0.1 & -6201 & -0.9 & 3100 & 0.1 & 0 & 0 & 0 & 0.8 \\
0 & 0 & 0 & 0 & 0 & 1 & 0 & 0 & 0 & 0 \\
0 & 0 & 3100 & 0.1 & -3100 & -0.1 & 0 & 0 & 0 & 0 \\
0 & 0 & 0 & 0 & 0 & 0 & 0 & 1 & 0 & 0 \\
0 & 0 & 0 & 0 & 0 & 0 & -3100 & -0.2 & 1550 & 0.1 \\
0 & 0 & 0 & 0 & 0 & 0 & 0 & 0 & 0 & 1 \\
0 & 0 & 0 & 0.8 & 0 & 0 & 1550 & 0.1 & -1550 & -0.9
\end{array}\right] \\
& B=10^{-6} \times\left[\begin{array}{ccccc}
0 & 0 & 0 & 0 & 0 \\
0.7752 & 0 & 0 & 0 & 0 \\
0 & 0 & 0 & 0 & 0 \\
0 & 0.7752 & 0 & 0 & 0 \\
0 & 0 & 0 & 0 & 0 \\
0 & 0 & 0.7752 & 0 & 0 \\
0 & 0 & 0 & 0 & 0 \\
0 & 0 & 0 & 0.7752 & 0 \\
0 & 0 & 0 & 0 & 0 \\
0 & 0 & 0 & 0 & 0.7752
\end{array}\right] \\
& E=[0,-1,0,-1,0,-1,0,-1,0,-1]^{T} \text {, } \\
& C_{y}=\left[\begin{array}{rrrrrrrrrr}
1 & 0 & 0 & 0 & 0 & 0 & 0 & 0 & 0 & 0 \\
-1 & 0 & 1 & 0 & 0 & 0 & 0 & 0 & 0 & 0 \\
0 & 0 & -1 & 0 & 1 & 0 & 0 & 0 & 0 & 0 \\
0 & 0 & 0 & 0 & 0 & 0 & 1 & 0 & 0 & 0 \\
0 & 0 & 0 & 0 & 0 & 0 & -1 & 0 & 1 & 0
\end{array}\right] .
\end{aligned}
$$

It should be noted that the output matrix $C_{y}$ extracts the inter-story drifts of the buildings, that is,

$$
y(t)=\left[y_{1}^{(l)}(t), y_{2}^{(l)}(t), y_{3}^{(l)}(t), y_{1}^{(r)}(t), y_{2}^{(r)}(t)\right]^{T},
$$

where $y_{1}^{(l)}=q_{1}^{(l)}, y_{i}^{(l)}=q_{i+1}^{(l)}-q_{i}^{(l)}, i=1,2, y_{1}^{(r)}=q_{1}^{(r)}, y_{2}^{(r)}=$ $q_{2}^{(r)}-q_{1}^{(r)}$. The matrices in (11) correspond to the following particular values of the mass, damping and stiffness coefficients: $\quad m_{i}^{(l)}=m_{j}^{(r)}=1.29 \times 10^{6} \mathrm{Kg} ; \quad c_{i}^{(l)}=c_{j}^{(r)}=10^{5} \mathrm{~N} \mathrm{~s} / \mathrm{m}$; $k_{i}^{(l)}=4 \times 10^{9} \mathrm{~N} / \mathrm{m} ; \quad k_{j}^{(r)}=2 \times 10^{9} \mathrm{~N} / \mathrm{m} ; \quad c_{1}^{(d)}=0, \quad c_{2}^{(d)}=10^{6} \mathrm{~N} \mathrm{~s} / \mathrm{m} ;$ $k_{j}^{(d)}=0$, for $i=1,2,3, j=1,2$. A detailed derivation of the firstorder state-space model can be found in [6].

\section{INCLUSION PRINCIPLE}

In this section, the definition of the Inclusion Principle together with the design of overlapping controllers are briefly presented. A rigorous treatment can be found in [9], [10], [11], [12], [13].

Consider a pair of linear systems

$$
\begin{aligned}
\mathbf{S}: \dot{x}(t) & =A x(t)+B u(t), & \tilde{\mathbf{S}}: \dot{\tilde{x}}(t) & =\tilde{A} \tilde{x}(t)+\tilde{B} \tilde{u}(t), \\
y(t) & =C_{y} x(t), & \tilde{y}(t) & =\tilde{C}_{y} \tilde{x}(t),
\end{aligned}
$$


where $x(t) \in \mathbb{R}^{\mathbf{n}}, u(t) \in \mathbb{R}^{\mathbf{m}}, y(t) \in \mathbb{R}^{1}$ are the state, the input, and the output of $\mathbf{S}$ at time $t \geq 0$ and $\tilde{x}(t) \in \mathbb{R}^{\tilde{\mathbf{n}}}, \tilde{u}(t) \in \mathbb{R}^{\tilde{\mathbf{m}}}$, $\tilde{y}(t) \in \mathbb{R}^{\tilde{1}}$ are the state, the input, and the output of $\tilde{\mathbf{S}} . A$, $B, C_{y}$ and $\tilde{A}, \tilde{B}, \tilde{C}_{y}$ are $n \times n, n \times m, l \times n$ and $\tilde{n} \times \tilde{n}, \tilde{n} \times \tilde{m}$, $\tilde{l} \times \tilde{n}$ dimensional matrices, respectively. Suppose that the dimensions of the state, the input, and the output vectors $x(t), u(t), y(t)$ of $\mathbf{S}$ are smaller than those of $\tilde{x}(t), \tilde{u}(t), \tilde{y}(t)$ of $\tilde{\mathbf{S}}$. Let $x\left(t ; x_{0}, u\right)$ and $y[x(t)]$ denote the state behavior and the corresponding output of $\mathbf{S}$ for a fixed input $u(t)$ and for an initial state $x(0)=x_{0}$, respectively. Similar notations $\tilde{x}\left(t ; \tilde{x}_{0}, \tilde{u}\right)$ and $\tilde{y}[\tilde{x}(t)]$ are used for the state behavior and output of the system $\tilde{\mathbf{S}}$.

Let us consider the following linear transformations:

$$
\begin{array}{ll}
V: \mathbb{R}^{\mathbf{n}} \longrightarrow \mathbb{R}^{\tilde{\mathbf{n}}}, & U: \mathbb{R}^{\tilde{\mathbf{n}}} \longrightarrow \mathbb{R}^{\mathbf{n}}, \\
R: \mathbb{R}^{\mathbf{m}} \longrightarrow \mathbb{R}^{\tilde{\mathbf{m}}}, & Q: \mathbb{R}^{\tilde{\mathbf{m}}} \longrightarrow \mathbb{R}^{\mathbf{m}}, \\
T: \mathbb{R}^{1} \longrightarrow \mathbb{R}^{\tilde{\mathbf{1}}}, & S: \mathbb{R}^{\tilde{\mathbf{1}}} \longrightarrow \mathbb{R}^{1},
\end{array}
$$

where $\operatorname{rank}(V)=n, \operatorname{rank}(R)=m, \operatorname{rank}(T)=l$ and such that $U V=I_{n}, Q R=I_{m}, S T=I_{l}$, where $I_{n}, I_{m}, I_{l}$ are the identity matrices of indicated dimensions.

Definition 1: (Inclusion Principle) A system $\tilde{\mathbf{S}}$ includes the system $\mathbf{S}$ if there exists a quadruplet of matrices $(U, V, R, S)$ such that, for any initial state $x_{0}$ and any fixed input $u(t)$ of $\mathbf{S}$, the choice

$$
\begin{aligned}
\tilde{x}_{0} & =V x_{0}, \\
\tilde{u}(t) & =R u(t), \text { for all } t \geq 0
\end{aligned}
$$

of the initial state $\tilde{x}_{0}$ and input $\tilde{u}(t)$ of the system $\tilde{\mathbf{S}}$, implies

$$
\begin{aligned}
x\left(t ; x_{0}, u\right) & =U \tilde{x}\left(t ; \tilde{x}_{0}, \tilde{u}\right), \\
y[x(t)] & =S \tilde{y}[\tilde{x}(t)], \text { for all } t \geq 0 .
\end{aligned}
$$

Suppose that the pairs of matrices $(U, V),(Q, R)$ and $(S, T)$ are given. Then, the expanded matrices $\tilde{A}, \tilde{B}$, and $\tilde{C}_{y}$ can be expressed as

$$
\tilde{A}=V A U+M, \quad \tilde{B}=V B Q+N, \quad \tilde{C}_{y}=T C_{y} U+L,
$$

where $M, N$ and $L$ are complementary matrices of appropriate dimensions. In terms of complementary matrices, the inclusion principle can be established in the following way.

Theorem 1: A system $\tilde{\mathbf{S}}$ includes the system $\mathbf{S}$ if and only if $U M^{i} V=0, U M^{i-1} N R=0, S L M^{i-1} V=0$ and $S L M^{i-1} N R=0$ for all $i=1,2, \ldots, \tilde{n}$.

Two particular forms of the inclusion principle, called restrictions and aggregations, are normally used in practice [9]. In this paper, a restriction has been chosen.

Proposition 1: A system $\mathbf{S}$ is a restriction of the system $\tilde{\mathbf{S}}$ if and only if $M V=0, N R=0$ and $L V=0$.

Let us suppose that the system $\mathbf{S}$ given in (13) admits an overlapping decomposition. In terms of the system matrices, this assumption means that $A, B$ and $C_{y}$ present a block tridiagonal structure

$$
\begin{aligned}
& A=\left[\begin{array}{cc:c}
A_{11} & A_{12} & 0 \\
A_{21} & A_{22} & A_{23} \\
\hdashline 0 & A_{32} & A_{33}
\end{array}\right], \quad B=\left[\begin{array}{cc:c}
B_{11} & B_{12} & 0 \\
B_{21} & B_{22} & B_{23} \\
\hdashline 0 & B_{32} & B_{33}
\end{array}\right], \\
& C_{y}=\left[\begin{array}{c:c:c}
\left(C_{y}\right)_{11} & \left(C_{y}\right)_{12} & 0 \\
\left(C_{y}\right)_{21} & \left(C_{y}\right)_{22} & - \\
\hdashline 0 & \left(C_{y}\right) & C_{23} \\
\hdashline & \left(C_{y}\right)_{33}
\end{array}\right],
\end{aligned}
$$

where $A_{i i}, B_{i j},\left(C_{y}\right)_{i j}, i, j=1,2,3$, are $n_{i} \times n_{i}, n_{i} \times m_{j}, l_{i} \times n_{j}$ dimensional matrices, respectively. The partition of the state $x=\left(x_{1}^{T}, x_{2}^{T}, x_{3}^{T}\right)^{T}$ has components of respective dimensions $n_{1}, n_{2}, n_{3}$, satisfying $n_{1}+n_{2}+n_{3}=n$; the partition of $u=\left(u_{1}^{T}, u_{2}^{T}, u_{3}^{T}\right)^{T}$ has components of dimensions $m_{1}, m_{2}, m_{3}$, such that $m_{1}+m_{2}+m_{3}=m$; and $y=\left(y_{1}^{T}, y_{2}^{T}, y_{3}^{T}\right)^{T}$ has components of respective dimensions $l_{1}, l_{2}, l_{3}$, satisfying $l_{1}+l_{2}+l_{3}=l$.

The controller design starts with the selection of the expansion transformations

$$
V=\left[\begin{array}{ccc}
I_{n_{1}} & 0 & 0 \\
0 & I_{n_{2}} & 0 \\
0 & I_{n_{2}} & 0 \\
0 & 0 & I_{n_{3}}
\end{array}\right], R=\left[\begin{array}{ccc}
I_{m_{1}} & 0 & 0 \\
0 & I_{m_{2}} & 0 \\
0 & I_{m_{2}} & 0 \\
0 & 0 & I_{m_{3}}
\end{array}\right], T=\left[\begin{array}{ccc}
I_{l_{1}} & 0 & 0 \\
0 & I_{l_{2}} & 0 \\
0 & I_{l_{2}} & 0 \\
0 & 0 & I_{l_{3}}
\end{array}\right]
$$

with their corresponding pseudoinverse contractions

$$
\begin{aligned}
U & =\left[\begin{array}{cccc}
I_{n_{1}} & 0 & 0 & 0 \\
0 & \frac{1}{2} I_{n_{2}} & \frac{1}{2} I_{n_{2}} & 0 \\
0 & 0 & 0 & I_{n_{3}}
\end{array}\right], \quad Q=\left[\begin{array}{cccc}
I_{m_{1}} & 0 & 0 & 0 \\
0 & \frac{1}{2} I_{m_{2}} & \frac{1}{2} I_{m_{2}} & 0 \\
0 & 0 & 0 & I_{m_{3}}
\end{array}\right], \\
S & =\left[\begin{array}{cccc}
I_{l_{1}} & 0 & 0 & 0 \\
0 & \frac{1}{2} I_{l_{2}} & \frac{1}{2} I_{l_{2}} & 0 \\
0 & 0 & 0 & I_{l_{3}}
\end{array}\right],
\end{aligned}
$$

where $\quad U=\left(V^{T} V\right)^{-1} V^{T}, \quad Q=\left(R^{T} R\right)^{-1} R^{T}, \quad S=\left(T^{T} T\right)^{-1} T^{T}$. Then, the expanded matrices $\bar{A}=V A U, \bar{B}=V B Q, \bar{C}_{y}=T C_{y} U$, have the form

$$
\begin{aligned}
\bar{A} & =\left[\begin{array}{cc:cc}
A_{11} & \frac{1}{2} A_{12} & \frac{1}{2} A_{12} & 0 \\
A_{21} & \frac{1}{2} A_{22} & \frac{1}{2} A_{22} & A_{23} \\
\hdashline A_{21} & \frac{1}{2} A_{22} & \frac{1}{2} A_{22} & A_{23} \\
0 & \frac{1}{2} A_{32} & \frac{1}{2} A_{32} & A_{33}
\end{array}\right], \bar{B}=\left[\begin{array}{cc:cc}
B_{11} & \frac{1}{2} B_{12} & \frac{1}{2} B_{12} & 0 \\
B_{21} & \frac{1}{2} B_{22} & \frac{1}{2} B_{22} & B_{23} \\
\hdashline B_{21} & \frac{1}{2} B_{22} & \frac{1}{2} B_{22} & B_{23} \\
0 & \frac{1}{2} B_{32} & \frac{1}{2} B_{32} & B_{33}
\end{array}\right] \\
\bar{C}_{y} & =\left[\begin{array}{cc:cc}
\left(C_{y}\right)_{11} & \frac{1}{2}\left(C_{y}\right)_{12} & \frac{1}{2}\left(C_{y}\right)_{12} & 0 \\
\left(C_{y}\right)_{21} & \frac{1}{2}\left(C_{y}\right)_{22} & \frac{1}{2}\left(C_{y}\right)_{22}\left(C_{y}\right)_{23} \\
\hdashline\left(C_{y}\right)_{21} & \frac{1}{2}\left(C_{y}\right)_{22} & \frac{1}{2}\left(C_{y}\right)_{22} & \left(C_{y}\right)_{23} \\
0 & \frac{1}{2}\left(C_{y}\right)_{32} & \frac{1}{2}\left(C_{y}\right)_{32} & \left(C_{y}\right)_{33}
\end{array}\right] .
\end{aligned}
$$

In order to get an almost-decoupled expanded system, we add complementary matrices as indicated in (17). In the case of a restriction, these matrices have the form

$$
\begin{aligned}
M & =\left[\begin{array}{rrrr}
0 & \frac{1}{2} A_{12} & -\frac{1}{2} A_{12} & 0 \\
0 & \frac{1}{2} A_{22} & -\frac{1}{2} A_{22} & 0 \\
0 & -\frac{1}{2} A_{22} & \frac{1}{2} A_{22} & 0 \\
0 & -\frac{1}{2} A_{32} & \frac{1}{2} A_{32} & 0
\end{array}\right], \quad N=\left[\begin{array}{rrrr}
0 & \frac{1}{2} B_{12} & -\frac{1}{2} B_{12} & 0 \\
0 & \frac{1}{2} B_{22} & -\frac{1}{2} B_{22} & 0 \\
0 & -\frac{1}{2} B_{22} & \frac{1}{2} B_{22} & 0 \\
0 & -\frac{1}{2} B_{32} & \frac{1}{2} B_{32} & 0
\end{array}\right], \\
L & =\left[\begin{array}{rrrr}
0 & \frac{1}{2}\left(C_{y}\right)_{12} & -\frac{1}{2}\left(C_{y}\right)_{12} & 0 \\
0 & \frac{1}{2}\left(C_{y}\right)_{22} & -\frac{1}{2}\left(C_{y}\right)_{22} & 0 \\
0 & -\frac{1}{2}\left(C_{y}\right)_{22} & \frac{1}{2}\left(C_{y}\right)_{22} & 0 \\
0 & -\frac{1}{2}\left(C_{y}\right)_{32} & \frac{1}{2}\left(C_{y}\right)_{32} & 0
\end{array}\right],
\end{aligned}
$$


resulting

$$
\begin{gathered}
\tilde{A}=\bar{A}+M=\left[\begin{array}{ll}
\tilde{A}_{11} & \tilde{A}_{12} \\
\tilde{A}_{21} & \tilde{A}_{22}
\end{array}\right]=\left[\begin{array}{cc:cc}
A_{11} & A_{12} & 0 & 0 \\
A_{21} & A_{22} & 0 & A_{23} \\
\hdashline A_{21} & 0 & A_{22} & A_{23} \\
0 & 0 & A_{32} & A_{33}
\end{array}\right], \\
\tilde{B}=\bar{B}+N=\left[\begin{array}{ll}
\tilde{\tilde{B}}_{11} & \tilde{B}_{12} \\
\tilde{B}_{21} & \tilde{B}_{22}
\end{array}\right]=\left[\begin{array}{cc:cc}
B_{11} & B_{12} & 0 & 0 \\
B_{21} & B_{22} & 0 & B_{23} \\
\hdashline B_{21} & 0 & B_{22} & B_{23} \\
0 & 0 & B_{32} & B_{33}
\end{array}\right], \\
\tilde{C}_{y}=\bar{C}_{y}+L=\left[\begin{array}{ll}
\left(\tilde{C}_{y}\right)_{11} & \left(\tilde{C}_{y}\right)_{12} \\
\left(\tilde{C}_{y}\right)_{21} & \left(\tilde{C}_{y}\right)_{22}
\end{array}\right]=\left[\begin{array}{cccc}
\left(C_{y}\right)_{11} & \left(C_{y}\right)_{12} & 0 & 0 \\
\left(C_{y}\right)_{21} & \left(C_{y}\right)_{22} & 0 & \left(C_{y}\right)_{23} \\
\hdashline\left(C_{y}\right)_{21} & 0 & \left(C_{y}\right)_{22} & \left(C_{y}\right)_{23} \\
0 & 0 & \left(C_{y}\right)_{32} & \left(C_{y}\right)_{33}
\end{array}\right] .
\end{gathered}
$$

The expanded system can be denoted by

$$
\begin{aligned}
\tilde{\mathbf{S}}: \dot{\tilde{x}}(t) & =\tilde{A} \tilde{x}(t)+\tilde{B} \tilde{u}(t), \\
\tilde{y}(t) & =\tilde{C}_{y} \tilde{x}(t),
\end{aligned}
$$

with state $\tilde{x}^{T}=\left(x_{1}^{T}, x_{2}^{T}, x_{2}^{T}, x_{3}^{T}\right)$, input $\tilde{u}^{T}=\left(u_{1}^{T}, u_{2}^{T}, u_{2}^{T}, u_{3}^{T}\right)$, and output $\tilde{y}^{T}=\left(y_{1}^{T}, y_{2}^{T}, y_{2}^{T}, y_{3}^{T}\right)$. Using the block notation given in (23), we can write

$$
\begin{aligned}
\tilde{\mathbf{S}}_{1}: & \dot{\tilde{x}}_{1}(t)=\tilde{A}_{11} \tilde{x}_{1}(t)+\tilde{B}_{11} \tilde{u}_{1}(t)+\tilde{A}_{12} \tilde{x}_{2}(t)+\tilde{B}_{12} \tilde{u}_{2}(t), \\
\tilde{y}_{1}(t) & =\left(\tilde{C}_{y}\right)_{11} \tilde{x}_{1}(t)+\left(\tilde{C}_{y}\right)_{12} \tilde{x}_{2}(t), \\
\tilde{\mathbf{S}}_{2}: & \dot{\tilde{x}}_{2}(t)=\tilde{A}_{22} \tilde{x}_{2}(t)+\tilde{B}_{22} \tilde{u}_{2}(t)+\tilde{A}_{21} \tilde{x}_{1}(t)+\tilde{B}_{21} \tilde{u}_{1}(t), \\
\tilde{y}_{2}(t) & =\left(\tilde{C}_{y}\right)_{21} \tilde{x}_{1}(t)+\left(\tilde{C}_{y}\right)_{22} \tilde{x}_{2}(t),
\end{aligned}
$$

where $\tilde{x}_{1}^{T}=\left(x_{1}^{T}, x_{2}^{T}\right), \tilde{u}_{1}^{T}=\left(u_{1}^{T}, u_{2}^{T}\right), \tilde{y}_{1}^{T}=\left(y_{1}^{T}, y_{2}^{T}\right), \tilde{x}_{2}^{T}=\left(x_{2}^{T}, x_{3}^{T}\right)$, $\tilde{u}_{2}^{T}=\left(u_{2}^{T}, u_{3}^{T}\right), \tilde{y}_{2}^{T}=\left(y_{2}^{T}, y_{3}^{T}\right)$. By removing the interconnection blocks, two decoupled expanded subsystems result

$$
\begin{aligned}
\tilde{\mathbf{S}}_{\mathbf{D}}^{(1)}: & \dot{\tilde{x}}_{1}(t)=\tilde{A}_{11} \tilde{x}_{1}(t)+\tilde{B}_{11} \tilde{u}_{1}(t), \\
\tilde{y}_{1}(t) & =\left(\tilde{C}_{y}\right)_{11} \tilde{x}_{1}(t), \\
\tilde{\mathbf{S}}_{\mathbf{D}}^{(2)}: & \dot{\tilde{x}}_{2}(t)=\tilde{A}_{22} \tilde{x}_{2}(t)+\tilde{B}_{22} \tilde{u}_{2}(t), \\
\tilde{y}_{2}(t) & =\left(\tilde{C}_{y}\right)_{22} \tilde{x}_{2}(t),
\end{aligned}
$$

which define a decoupled expanded system

$$
\begin{aligned}
\tilde{\mathbf{S}}_{\mathbf{D}}: \dot{\tilde{x}}(t) & =\tilde{A}_{D} \tilde{x}(t)+\tilde{B}_{D} \tilde{u}(t), \\
\tilde{y}(t) & =\left(\tilde{C}_{y}\right)_{D} \tilde{x}(t),
\end{aligned}
$$

where $\tilde{A}_{D}, \tilde{B}_{D},\left(\tilde{C}_{y}\right)_{D}$ are block diagonal, $\tilde{A}_{D}=\operatorname{diag}\left\{\tilde{A}_{11}, \tilde{A}_{22}\right\}$, $\tilde{B}_{D}=\operatorname{diag}\left\{\tilde{B}_{11}, \tilde{B}_{22}\right\}$ and $\left(\tilde{C}_{y}\right)_{D}=\operatorname{diag}\left\{\left(\tilde{C}_{y}\right)_{11},\left(\tilde{C}_{y}\right)_{22}\right\}$. At this point, a expanded decentralized controller $\tilde{u}_{D}(t)=\tilde{K}_{D} \tilde{x}(t)$ for $\tilde{\mathbf{S}}_{\mathrm{D}}$ can be designed by independently computing local controllers for $\tilde{\mathbf{S}}_{\mathbf{D}}^{(1)}$ and $\tilde{\mathbf{S}}_{\mathbf{D}}^{(2)}$.

Definition 2: (Contractibility) Suppose that $\tilde{\mathbf{S}}$ is an expansion of the system $\mathbf{S}$. Then, a control law $\tilde{u}(t)=\tilde{K} \tilde{x}(t)$ for $\tilde{\mathbf{S}}$ is contractible to the control law $u(t)=K x(t)$ for $\mathbf{S}$ if there exist transformations as in (14) such that, for any initial state $x_{0} \in \mathbb{R}^{\mathrm{n}}$ and any input $u(t) \in \mathbb{R}^{\mathrm{m}}$, if $\tilde{x}_{0}=V x_{0}$ and $\tilde{u}(t)=R u(t)$ then $K x\left(t ; x_{0}, u\right)=Q \tilde{K} \tilde{x}\left(t ; V x_{0}, R u\right)$ for all $t \geq 0$.
Proposition 2: Suppose that $\tilde{\mathbf{S}}$ is an expansion of the system $\mathbf{S}$. Then, a control law $\tilde{u}(t)=\tilde{K} \tilde{x}(t)$ for $\tilde{\mathbf{S}}$ is contractible to the control law $u(t)=K x(t)$ for $\mathbf{S}$ if and only if $Q \tilde{K} V=K$, $Q \tilde{K} M^{i} V=0, Q \tilde{K} M^{i-1} N R=0$, for $i=1, \ldots, \tilde{n}$.

According to Definition 2 and Proposition 2, the expanded decentralized controller $\tilde{u}_{D}(t)=\tilde{K}_{D} \tilde{x}(t)$ can be contracted to an overlapping controller $u(t)=K_{o} x(t)$ to be implemented into the original system $\mathbf{S}$. The contracted gain matrix is computed as

$$
K_{o}=Q \tilde{K}_{D} V=\left[\begin{array}{cc:c}
K_{11} & K_{12} & 0 \\
K_{21} & K_{22} & K_{23} \\
\hdashline 0 & K_{32} & K_{33} \\
& &
\end{array}\right],
$$

which has a desired block tridiagonal structure.

\section{Decentralized Controllers Design}

In this paper, the independent local controllers for $\tilde{\mathbf{S}}_{\mathbf{D}}^{(1)}$ and $\tilde{\mathbf{S}}_{\mathbf{D}}^{(2)}$ have been designed as optimal LQR controllers; however, it is clear that other control strategies could have been used. More precisely, for the expanded decoupled subsystems $\tilde{\mathbf{S}}_{\mathbf{D}}^{(1)}$ and $\tilde{\mathbf{S}}_{\mathbf{D}}^{(2)}$, let us consider the local quadratic cost functions

$$
\begin{aligned}
& \tilde{J}_{D}^{(1)}\left(\tilde{x}_{10}, \tilde{u}_{1}(t)\right)=\int_{0}^{\infty}\left[\tilde{x}_{1}^{T}(t) \tilde{Q}_{1}^{*} \tilde{x}_{1}(t)+\tilde{u}_{1}^{T}(t) \tilde{R}_{1}^{*} \tilde{u}_{1}(t)\right] d t, \\
& \tilde{J}_{D}^{(2)}\left(\tilde{x}_{20}, \tilde{u}_{2}(t)\right)=\int_{0}^{\infty}\left[\tilde{x}_{2}^{T}(t) \tilde{Q}_{2}^{*} \tilde{x}_{2}(t)+\tilde{u}_{2}^{T}(t) \tilde{R}_{2}^{*} \tilde{u}_{2}(t)\right] d t,
\end{aligned}
$$

where $\tilde{x}_{10}$ and $\tilde{x}_{20}$ are the initial states of $\tilde{\mathbf{S}}_{\mathbf{D}}^{(1)}$ and $\tilde{\mathbf{S}}_{\mathbf{D}}^{(2)}$, respectively, and $\tilde{Q}_{1}^{*}, \tilde{Q}_{2}^{*}, \tilde{R}_{1}^{*}$ and $\tilde{R}_{2}^{*}$ are appropriate expanded matrices. The gain matrices for the control laws that minimize the cost functions given in (29)

$$
\tilde{u}_{1}(t)=\tilde{K}_{1} \tilde{x}_{1}(t), \quad \tilde{u}_{2}(t)=\tilde{K}_{2} \tilde{x}_{2}(t),
$$

can be independently computed as

$$
\tilde{K}_{1}=\left[\tilde{R}_{1}^{*}\right]^{-1} \tilde{B}_{1}^{T} \tilde{P}_{1}, \quad \tilde{K}_{2}=\left[\tilde{R}_{2}^{*}\right]^{-1} \tilde{B}_{2}^{T} \tilde{P}_{2},
$$

where $\tilde{P}_{1}$ and $\tilde{P}_{2}$ are the solutions of the corresponding Riccati equations. In the decoupled expanded system $\tilde{\mathbf{S}}_{\mathbf{D}}$, the gain matrix of the controller $\tilde{u}(t)=\tilde{K}_{D} \tilde{x}(t)$ which minimizes the cost function

$$
\tilde{J}_{D}\left(\tilde{x}_{0}, \tilde{u}(t)\right)=\int_{0}^{\infty}\left[\tilde{x}^{T}(t) \tilde{Q}_{D}^{*} \tilde{x}(t)+\tilde{u}^{T}(t) \tilde{R}_{D}^{*} \tilde{u}(t)\right] d t,
$$

with $\tilde{Q}_{D}^{*}=\operatorname{diag}\left\{\tilde{Q}_{1}^{*}, \tilde{Q}_{2}^{*}\right\}$ and $\tilde{R}_{D}^{*}=\operatorname{diag}\left\{\tilde{R}_{1}^{*}, \tilde{R}_{2}^{*}\right\}$, can be written as a block diagonal gain matrix $\tilde{K}_{D}^{*}=\operatorname{diag}\left\{\tilde{K}_{1}^{*}, \tilde{K}_{2}^{*}\right\}$. Finally, the expanded decentralized controller $\tilde{u}_{D}(t)=\tilde{K}_{D} \tilde{x}(t)$ is contracted to an overlapping controller $u(t)=K_{o} x(t)$ to be implemented into the original system $\mathbf{S}$. The contracted gain matrix is computed as

$$
K_{o}=Q \tilde{K}_{D} V
$$

which has the desired block tridiagonal structure shown in (28). 


\section{CONTROLLER DESIGN}

In this section, a centralized controller and a decentralized overlapping controller are designed for the system (9) defined by the matrices given in (11). In the overall coupled model, the buildings are linked by a single damper, with damping constant $c_{2}^{(d)}=10^{6} \mathrm{~N} \mathrm{~s} / \mathrm{m}$, located at the second story. This can be considered as a minimal configuration of the passive control system, in the sense that the numerical simulations show that no significant reduction of the vibrational response results when a similar second damper is placed linking the first stories, or elastic linking elements are considered. In terms of the damping and stiffness coefficients, this means $c_{1}^{(d)}=0, k_{1}^{(d)}=k_{2}^{(d)}=0$ (see Fig. 1).

\section{Centralized State-Feedback Controller}

The centralized LQR controller is computed using the overall coupled model and the cost matrices $Q^{*}=C_{y}^{T} C_{y}$, $R^{*}=10^{-17} \times I_{5}$, which define the quadratic cost function

$$
\begin{aligned}
J\left(x_{0}, u(t)\right) & =\int_{0}^{\infty}\left[x^{T}(t) Q^{*} x(t)+u^{T}(t) R^{*} u(t)\right] d t, \\
& =\int_{0}^{\infty}\left[y^{T}(t) y(t)+u^{T}(t) R^{*} u(t)\right] d t .
\end{aligned}
$$

The resulting gain matrix is

$K_{c}=10^{7} \times$

$\left[\begin{array}{rrrrrrrrrr}1.140 & 0.522 & 0.325 & 0.022 & -0.221 & -0.013 & -0.075 & 0.003 & 0.091 & 0.016 \\ -0.218 & 0.022 & 1.459 & 0.509 & -0.109 & 0.009 & -0.073 & 0.012 & 0.159 & 0.025 \\ 0.115 & -0.013 & 0.096 & 0.009 & 1.139 & 0.531 & 0.551 & 0.021 & -0.296 & 0.026 \\ 0.107 & 0.003 & 0.203 & 0.012 & -0.340 & 0.021 & 2.267 & 0.744 & 0.204 & -0.010 \\ 0.059 & 0.016 & -0.834 & 0.025 & 0.565 & 0.026 & -0.451 & -0.010 & 2.686 & 0.732\end{array}\right]$.

The gain matrix $K_{C}$ is a full matrix and, consequently, to compute the control action for any actuator, the knowledge of the complete state in both buildings is required.

\section{Decentralized Overlapping Controller}

For the decentralized controller, we follow a two-level decentralized approach. At the first level, the linking elements are ignored, and a controller is independently designed for each building. At the second level, an overlapping controller is designed for the left building, which is considered as a large building. More precisely, we consider the left building as composed by two overlapping subsystems $\tilde{\mathbf{S}}_{\mathbf{D}}^{(1)}=[1,2]$ (first and second story) and $\tilde{\mathbf{S}}_{\mathbf{D}}^{(2)}=[2,3]$ (second and third story), with an overlapped part in the second story. In the expanded space, the following matrices are used:

$$
\begin{aligned}
\left(\tilde{C}_{y}\right)_{11} & =\left[\begin{array}{rrrr}
1 & 0 & 0 & 0 \\
-1 & 0 & 1 & 0
\end{array}\right], & \left(\tilde{C}_{y}\right)_{22} & =\left[\begin{array}{rrrr}
1 & 0 & 0 & 0 \\
-1 & 0 & 1 & 0
\end{array}\right], \\
\tilde{Q}_{1}^{*} & =\left(\tilde{C}_{y}\right)_{11}^{T}\left(\tilde{C}_{y}\right)_{11}, & \tilde{Q}_{2}^{*} & =\left(\tilde{C}_{y}\right)_{22}^{T}\left(\tilde{C}_{y}\right)_{22}, \\
\tilde{R}_{1}^{*} & =10^{-17} \times I_{2}, & \tilde{R}_{2}^{*} & =10^{-17} \times I_{2} .
\end{aligned}
$$

to compute a decentralized expanded LQR controller, which is subsequently contracted to obtain a local overlapping controller for the left building with block tridiagonal gain matrix $K_{o}^{(l)}$. Regarding to the right building, matrices $\left(Q^{*}\right)^{(r)}$ and $\left(R^{*}\right)^{(r)}$ with the same structure as those shown in (36) are used to independently design a local LQR controller with gain matrix $K^{(r)}$.The gain matrix for the overall system is finally obtained as the block diagonal matrix $K_{o}=\operatorname{diag}\left\{K_{o}^{(l)}, K^{(r)}\right\}$. In our particular example, we have

$K_{o}=10^{7} \times$

$\left[\begin{array}{cccccccccc}0.835 & 0.441 & 0.411 & -0.049 & 0 & 0 & 0 & 0 & 0 & 0 \\ -0.414 & -0.024 & 1.039 & 0.494 & -0.001 & 0.005 & 0 & 0 & 0 & 0 \\ 0 & 0 & -0.002 & 0.009 & 1.246 & 0.557 & 0 & 0 & 0 & 0 \\ 0 & 0 & 0 & 0 & 0 & 0 & 2.485 & 0.781 & -0.015 & 0.007 \\ 0 & 0 & 0 & 0 & 0 & 0 & -0.015 & 0.007 & 2.470 & 0.788\end{array}\right]$

The gain matrix $K_{o}$ is a structured matrix that allows to compute the control action for any actuator using only the local state of the corresponding building. Moreover, for the large building, the control actions can be computed using only the state of neighboring stories.

\section{NUMERICAL SIMULATIONS}

In this section, numerical simulations of the free and controlled responses of the two-building system, using the El Centro 1940 earthquake as acceleration input, are presented.
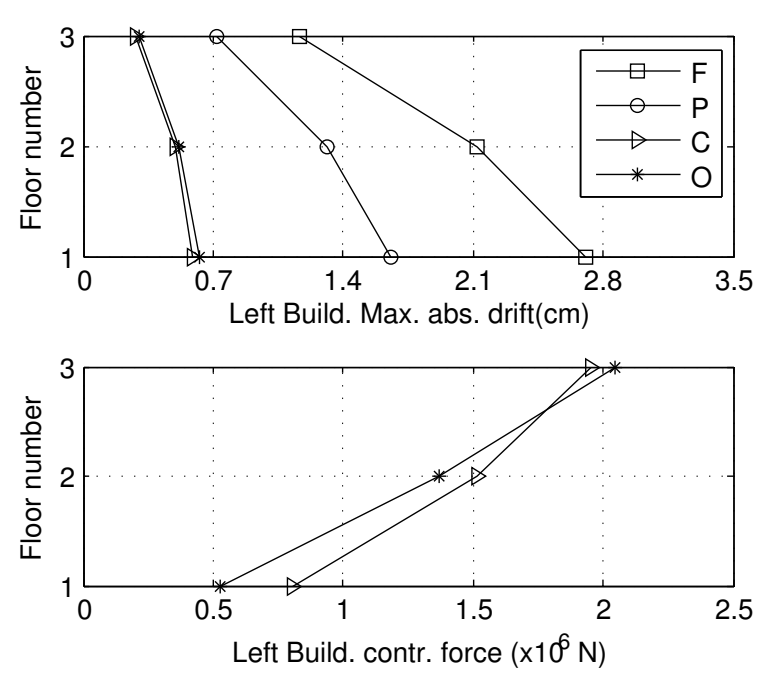

Fig. 2. Left building inter-story drifts and control forces

The maximum absolute inter-story drifts, and the maximum absolute control actions are displayed in Fig. 2 and Fig. 3. Four different situations are showed: (i) free response of the uncoupled system (denoted by $\mathrm{F}$ in the legend); (ii) free response of the coupled system, i.e., response under passive control (denoted by P); (iii) response of the coupled system under the centralized full-state feedback control (denoted by C); and (iv) response of the coupled system under the decentralized overlapping control (denoted by $\mathrm{O}$ ). The graphics show that a remarkable reduction of the vibrational response is achieved by the passive control system. Regarding the active controllers, the performance of the decentralized overlapping control is certainly excellent: despite the decentralized design and the restricted exchange of information, the decentralized controller behaves practically the same as the overall centralized controller.

The obtained results, the different characteristics of passive and active control elements, and the exceptional conditions under which a seismic protection control system must work, suggest the combination of both kinds of control elements to 

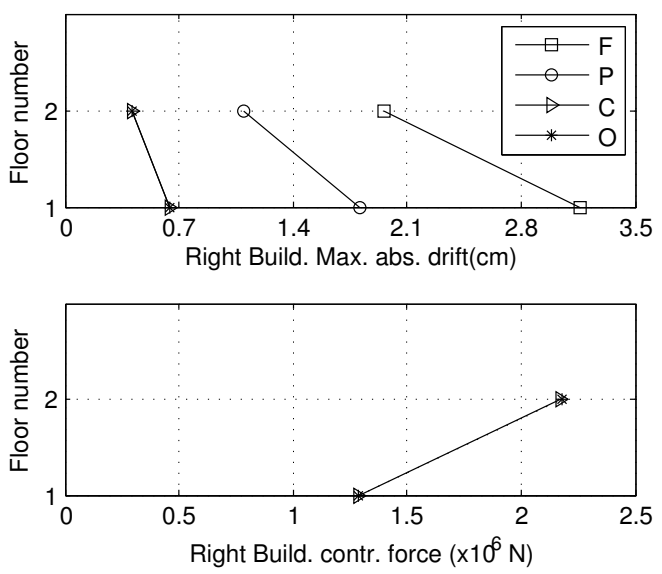

Fig. 3. Right building inter-story drifts and control forces

\begin{tabular}{|c|c|c|c||c|c|}
\cline { 2 - 6 } \multicolumn{1}{c|}{} & \multicolumn{3}{c||}{ Left Building } & \multicolumn{2}{c|}{ Right Building } \\
\cline { 2 - 6 } \multicolumn{1}{c|}{} & $y_{1}^{(l)}$ & $y_{2}^{(l)}$ & $y_{3}^{(l)}$ & $y_{1}^{(r)}$ & $y_{2}^{(r)}$ \\
\hline Free & 2.71 & 2.13 & 1.17 & 3.16 & 1.95 \\
\hline Passive & 1.65 & 1.32 & 0.72 & 1.81 & 1.10 \\
\hline Centralized & 0.59 & 0.49 & 0.28 & 0.65 & 0.42 \\
\hline Overlapping & 0.62 & 0.52 & 0.30 & 0.65 & 0.42 \\
\hline Left failure & 1.24 & 1.03 & 0.61 & 0.68 & 0.43 \\
\hline Right failure & 0.65 & 0.54 & 0.31 & 1.64 & 1.03 \\
\hline
\end{tabular}

Maximum absolute inter-story drifts $(\mathrm{cm})$

design an active-passive fault-tolerant decentralized control system. From this point of view, the free uncoupled response corresponds to a total failure mode; the free coupled response can be seen as a full failure of the active control system; partial failures of the active control system can be associated to the failure of the corresponding local active control system.

\begin{tabular}{|c|c|c|c||c|c|}
\cline { 2 - 6 } \multicolumn{1}{c|}{} & \multicolumn{3}{c||}{ Left Building } & \multicolumn{2}{c|}{ Right Building } \\
\cline { 2 - 6 } \multicolumn{1}{c|}{} & $u_{1}^{(l)}$ & $u_{2}^{(l)}$ & $u_{3}^{(l)}$ & $u_{1}^{(r)}$ & $u_{2}^{(r)}$ \\
\hline Centralized & 0.81 & 1.52 & 1.95 & 1.29 & 2.17 \\
\hline Overlapping & 0.52 & 1.37 & 2.04 & 1.28 & 2.18 \\
\hline Left failure & 0 & 0 & 0 & 1.35 & 2.28 \\
\hline Right failure & 0.54 & 1.42 & 2.12 & 0 & 0 \\
\hline
\end{tabular}

Maximum absolute actuation force $\left(\times 10^{6} \mathrm{~N}\right)$

Table I presents the maximum inter-story drifts for the different failure modes; the maximum absolute control actions are collected in Table II. These tables also include the data corresponding to the centralized controller as a reference. When the active control system is in full-failure mode, a remarkable reduction of the maximum inter-story drifts in both buildings is achieved by the passive control system. In case of semi-failure mode of the active control system, i.e., when one of the local active controllers fails, the building that remains actively controlled is not affected by the failure; moreover, the control forces in the working active controller increase slightly and act through the linking elements to drive the response of the failing building to a level that is clearly below the level obtained by the pure passive control. In all the cases, the results achieved by the decentralized overlapping controller are similar to those obtained by the centralized controller.

\section{CONCLUSIONS}

A combination of active and passive control elements have been used to design a decentralized control strategy suitable for wireless implementation in large adjacent buildings. Numerical simulations of the vibrational response of the system have been conducted, using the El Centro NS 1940 earthquake as ground acceleration. The simulation results indicate that the decentralized active controller, despite the decentralized design and the reduced exchange of information, behaves practically the same as the overall centralized active controller, and it is not affected by the failure of the passive control system. When the active control system is in full-failure mode, a remarkable reduction of the maximum inter-story drifts in both buildings is achieved by the passive control system. In case of semi-failure mode of the active control system, that is, when one of the local active controllers fails, the building that remains actively controlled is not affected by the failure; moreover, the control forces in the working active controller increase slightly and act through the linking elements to drive the response of the failing building to a level that is clearly below the level obtained by the pure passive control.

\section{REFERENCES}

[1] A. Bhaskararao and R. Jangid, "Seismic response of adjacent buildings connected with friction dampers," Bulletin of Earthquake Engineering, vol. 4, pp. 43-64, 2006.

[2] R. Christenson, B. Spencer, and E. Johnson, "Semiactive connected control method for adjacent multidegree-of-freedom buildings," Journal of Engineering Mechanics, vol. 133, no. 3, pp. 290-298, 2007.

[3] K. Seto, Y. Toba, and Y. Matsumoto, "Reduced order modelling and vibration control methods for flexible structures arranged in parallel," in Proceedings of the American Control Conference, Seattle, USA, 1995, pp. 2344-2348.

[4] Z. Yang, Y. Xu, and X. Lu, "Experimental seismic study of adjacent buildings with fluid dampers," Journal of Structural Engineering, vol. 129, no. 2, pp. 197-205, 2003.

[5] W. Zhang and Y. Xu, "Dynamic characteristics and seismic response of adjacent buildings linked by discrete dampers," Earthquake Engineering and Structural Dynamics, vol. 28, pp. 1163-1185, 1999.

[6] Y. Wang, J. Lynch, and K. Law, "Decentralized $H_{\infty}$ controller design for large-scale civil structures," Earthquake Engineering and Structural Dynamics, vol. 38, pp. 377-401, 2009.

[7] R. Swartz and J. Lynch, "Strategic network utilization in a wireless structural control system for seismically excited structures," Journal of Structural Engineering, pp. 597-608, 2009.

[8] F. Palacios-Quiñonero, J. Rodellar, and J. M. Rossell, "Sequential design of multi-overlapping controllers for longitudinal multioverlapping systems," Journal of Applied Mathematics and Computation, vol. 217, no. 3, pp. 1170-1183, 2010.

[9] D. Šiljak, Decentralized Control of Complex Systems. New York, USA: Academic Press, 1991.

[10] L. Bakule, J. Rodellar, and J. M. Rossell, "Generalized selection of complementary matrices in the inclusion principle," IEEE Transactions on Automatic Control, vol. 45, no. 6, pp. 1237-1243, 2000.

[11] _ , "Structure of expansion-contraction matrices in the inclusion principle for dynamic systems," SIAM J. Matrix Anal. Appl., vol. 21, no. 4, pp. 1136-1155, 2000.

[12] L. Bakule, J. Rodellar, J. M. Rossell, and P. Rubió, "Preservation of controllability-observability in expanded systems," IEEE Transactions on Automatic Control, vol. 46, no. 7, pp. 1155-1162, 2001.

[13] S. Stanković and D. Šiljak, "Contractibility of overlapping decentralized control," Systems \& Control Letters, vol. 44, pp. 189-199, 2001. 